\title{
I did eat my vegetables. Agreement between parent and child food intake diaries
}

\author{
Natalie Rangelov ${ }^{1, *}$, L Suzanne Suggs ${ }^{1,2}$ and Pedro Marques-Vidal ${ }^{3}$ \\ 'BeCHANGE Research Group, Institute for Public Communication (ICP), Faculty of Communication Sciences, \\ Università della Svizzera italiana, Via G. Buffi 13, 6900 Lugano, Switzerland: ${ }^{2}$ Institute of Global Health Innovation, \\ Faculty of Medicine, imperial College London, London, UK: ${ }^{3}$ Internal Medicine, Faculty of Biology and Medicine, \\ University of Lausanne, Lausanne University Hospital (CHUV), Lausanne, Switzerland
}

Submitted 8 January 2016: Final revision received 15 April 2016: Accepted 4 May 2016: First published online 5 July 2016

\begin{abstract}
Objective: To assess the level of agreement between children and their parents when reporting a child's food consumption.

Design: Cross-sectional study in which children and parents independently completed $7 \mathrm{~d}$ food diaries describing the foods and drinks the child consumed at every meal and snack. The association between child and parent reporting was assessed for nineteen food groups using Kendall's tau-b non-parametric correlations, Spearman's rank correlations, kappa coefficients and Lin's concordance measure of agreement. Results were also stratified by gender of the child and his/her grade at school.

Setting: Households in Ticino, Switzerland, April-June 2014.

Subjects: Two hundred and ninety-nine children aged 6-12 years and one of their parents participated, with 264 providing complete data (35\% completion rate).

Results: Results showed a high level of agreement between child and parent reporting. Spearman correlations ranged from 0.55 (sauces) and 0.57 (fatty meat) to $0 \cdot 80$ (fruit), 0.83 (starchy foods) and 0.84 (pastries). All nineteen Spearman correlations were significant at the 0.001 level. Kendall's tau-b correlations ranged from 0.44 (fat meat) to 0.81 (puff pastry). Kappa values showed low to high levels of agreement, ranging from 0.15 (sweets) to 0.77 (puff pastry). Lin's concordance correlation coefficients ranged from 0.39 (whole grains) to 0.86 (puff pastry).

Conclusions: When assessing the eating behaviour of children using a $7 \mathrm{~d}$ food diary, children's reports might be as reliable as their parents'.
\end{abstract}

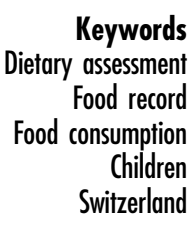

Childhood eating behaviours establish lifelong eating habits. Poor eating behaviour in childhood is a risk factor for overweight and obesity, cancer, type 2 diabetes and many other non-communicable conditions in adult$\operatorname{hood}^{(1)}$. Yet, measuring children's eating behaviour is riddled with challenges stemming from accuracy in recall, correct portion sizes, observation effects and participant burden $^{(2-6)}$.

Eating habits of children and adults are most commonly measured using FFQ, food diaries and $24 \mathrm{~h}$ recall methods ${ }^{(5)}$. While there are many variations, adults are often asked to indicate, from a list of items, the frequency of those foods consumed over the past 6 or 12 months. FFQ are most appropriate for epidemiological studies with large samples as they can help identify risk factors for disease and dietary patterns. Still, FFQ are limited by the variety of food items listed, accuracy in food consumption recall and the ability to accurately capture mixed foods (i.e. lasagne) $^{(7,8)}$.
Food diaries are thought to increase accuracy of reporting as they are completed daily. They are typically recommended for a motivated population, due to the burden of completing them ${ }^{(5,9)}$. However, some literature reports inaccuracies when comparing food records with biomarkers and suggest using a mix of assessment methods to collect more precise food intake data ${ }^{(4,5,10)}$. Twenty-four hour recall methods are brief and easy to administer, but do not allow for a description of a 'typical diet' as they represent a single day ${ }^{(7,8)}$. A study conducted in a sample of university students found that food diaries and $24 \mathrm{~h}$ recalls captured different information ${ }^{(11)}$. Another study found overall weaker associations of parent-child diet similarity when using FFQ than with food diaries ${ }^{(12)}$.

Children can face difficulty in recalling the foods that they consumed when the time gap between reporting and food consumption is long ${ }^{(5,9,13,14)}$. Additionally, in the age range from 6 to 10 years, their reading abilities can differ extensively and portion size estimation represents 
a common challenge $e^{(5,9,13,15)}$. While direct observation and clinical measures can serve as indicators of food consumption, they are not always feasible for public health practice and research. Hence, we are left with a conundrum on effective and efficient ways to measure children's food consumption outside controlled settings. One possible solution could be to ask children to report it directly, but little is known regarding their ability to accurately complete a food diary. There is also some disagreement on whether children or their parents should provide the information ${ }^{(5,9)}$. A review of studies assessing the validity of child/adolescent FFQ compared with biomarkers and/or other assessment method (i.e. $24 \mathrm{~h}$ recall) showed that studies where the FFQ were completed by the parents instead of children, and studies where FFQ were completed by children assisted by their parents or teacher, had weak to moderate validity ${ }^{(5)}$.

Studies measuring agreement between children and parents outside the food consumption domain show that children and parents often differ in their reporting of food insecurity ${ }^{(16)}$, health-related quality of life $^{(17)}$ and engagement in physical activity by children with disabilities $^{(18)}$. These studies suggest that parents are not always aware of children's precise attitudes and perceptions about their own health and health behaviours. As food consumption reporting may be less susceptible to perceptions, assuming portion sizes are not the object of measurement, we hypothesize that children and parents may both be able to report in an accurate way what a child eats, especially when the child eats in the presence of the parent. Hence, the purpose of the present paper was to assess the level of agreement between children and their parents when reporting a child's food consumption over a $7 \mathrm{~d}$ period using a food diary. A second aim was to assess the level of agreement between children and their parents according to children's gender and grade in school. As there is currently no validated measure for eating behaviour of children in Switzerland, a new instrument was developed.

\section{Methods}

\section{Study setting and sampling}

A cross-sectional study with children attending elementary and middle schools (aged 6-12 years) and one of their parents was conducted between April and June 2014. To be included, families (parent-child dyads) had to live in Ticino, Switzerland, and be able to complete the study in Italian. The Cantonal Department of Education, Culture and Sport (DECS) agreed to the study and identified four elementary schools and four middle schools that were representative of schools in Ticino. Directors of these schools were contacted and they agreed to include their school in the project. The directors recruited teachers and asked them to invite every child in their class, along with their parents, to participate in the study. They sent a package to each child-parent dyad that contained a letter describing the study, a consent form to be signed by the child's parent, two copies of the weekly food diary, a short questionnaire, and instructions with an example of how to complete the diary. Teachers also provided oral instructions to children on how to complete the diary and collected them at the end of the week of completion. The diaries were then sent back to the research team at the university.

The Ethics Committee of Canton Ticino reviewed the study and judged it exempt from full review. In accordance with the Helsinki Declaration, participants were fully informed of the study purpose and all parents provided their written informed consent before participating.

\section{Questionnaires and data collected}

The questionnaire collected the age and gender of the parent and the child, height and weight of the child, and the child's class at school. Children and parents separately completed a daily diary of foods and beverages the child consumed at breakfast, lunch and dinner, and during snack breaks (morning, afternoon and after dinner), every day for seven consecutive days. In Ticino, school lunches are not provided in most schools and the majority of children go home for lunch. As such, most meals were consumed primarily at home, excluding the morning snack, and parents were present during most of their child's meals. For each meal and snack time, the participant wrote exactly what the child ate in an open form text box on paper. No portion size was asked for given the known challenges of accurate reporting and that assessing portion size does not necessarily increase validity $^{(5)}$. Consistent with studies which suggest that children younger than 8 years of age face difficulties in estimating portions, and studies that found weak to moderate correlations when portions were assessed with children younger than 12 years of age $\mathrm{s}^{(5,13,19,20)}$, our study did not measure portion sizes.

Two versions of the food diary were used: one for elementary-school children and one for middle-school children. The versions differed only in the size of font and the space allowed for data entry. Middle-school children used a one-page diary for the full week, whereas children in elementary school reported each day on a separate page (see online supplementary material, Supplemental Figs 1 and 2). Parents were asked to not help the children recall what they ate and to help their children only if they needed assistance in understanding what the diary asked them to do and where to write their answers. Parents and children were asked to write 'I do not know' when they did not know or did not remember what was consumed. The importance of honest information and completing the diary after each meal and snack was stressed. 
Once the data were collected, three researchers independently coded the diaries. First, the food items were divided in groups according to the Swiss food pyramid ${ }^{(21)}$. A dietitian helped to develop subgroups of food items and mixed foods. A second coder then double-checked a random sample of food diaries. Food groups are shown in the online supplementary material, Supplemental Table 1. Food items were coded as dichotomous variables for each day and each meal $(0=$ did not eat a food from that (sub) group; $1=$ did eat a food from that (sub)group).

\section{Statistical analysis}

Statistical analyses were conducted using the statistical software package IBM SPSS Statistics Version 21.0. Descriptive results were expressed as number and frequency of participants for categorical variables and as range or mean and standard deviation for continuous variables. The weekly proportion of eating a certain food was computed for nineteen food groups consumed, using the total count (range 0-42 times per week) of each group consumed in a week and dividing it by the number of completed entries in a week (range 0-7d completed). Given that the number of meal occasions in which parents or children wrote 'I do not know' was very low, these data were coded and treated as missing data. The analyses in the present study were performed only among childrenparents pairs that completed the diary for all seven days.

Associations between children's and parents' results were assessed using Spearman's non-parametric correlations $(\rho)$, Lin's level of agreement statistics and Kendall's tau-b $\left(\tau_{\mathrm{B}}\right)$ non-parametric correlations. Spearman's rank correlations were used to test order and to compare with the results of the literature; $\rho$ values between $0 \cdot 10$ and 0.29 indicate a small correlation, between 0.30 and 0.49 a medium correlation, and between 0.50 and 1.00 a large correlation $^{(22)}$. Lin's statistics were used to test how well the two sets fit a $45^{\circ}$ line. As the algorithm assessing Spearman's correlation in IBM SPSS Statistics did not take into account tied values, Kendall's $\tau_{\mathrm{B}}$ correlations were computed, using the same methodology as Spearman's correlations, as they are appropriate to use with tied data ${ }^{(23)}$.

Consistent with Thiagarajah et al.'s validation study of a food intake questionnaire ${ }^{(13)}$, Cohen's kappa $(\kappa)$ was used to test the agreement between children's and parents' reporting of weekly consumption occasions. The lower bound of the $95 \% \mathrm{CI}$ of the $\kappa$ estimate was used to categorize agreement and the same categorization of $\kappa$ values as performed by Thiagarajah et al. ${ }^{(13)}$ was applied ${ }^{(24)}$ to allow comparison. Thus, $\kappa$ values between 0.20 and 0.45 indicate moderate agreement, $\kappa$ values higher than 0.45 and lower than 0.75 indicate substantial agreement, and $\kappa$ values equal to or higher than 0.75 indicate very good agreement $^{(13,24)}$. Finally, the level of agreement between children and their parents was analysed stratifying by gender and grade in school. Although no test was conducted to compare $\rho$ values by gender or school grade, the $95 \%$ CI of the $\kappa$ estimates are provided to allow for a 'side by side' comparison.

\section{Results}

\section{Sample characteristics}

Of the 745 children invited to participate by the teachers in eight classrooms, 299 consent forms, food diary pairs and questionnaires were returned to the research team ( $40 \%$ participation rate). After excluding children-parent pairs that did not complete the diaries for all seven days, a total of 264 children and parent pairs were included in the analysis $(35 \%$ of the families invited, $88 \%$ of the consenting parents). Fifty-two per cent of children were female and their age ranged between 7 and 14 years (mean 10.0 (SD 1.7) years). The percentage of children attending 1st, 2nd, 3rd, 4th and 5th grade of elementary school was 10, 11, 19, 23 and $15 \%$, respectively; $15 \%$ attended 1 st grade of middle school and $6 \%$ attended 2 nd grade. Participating parents were mostly female (95\%) and aged between 28 and 62 years (mean $41 \cdot 2$ (SD 5.3) years).

\section{Association between children's and parents' reporting}

Food consumption reported by children and their parents, and the proportion of consumption at meals, are summarized in the online supplementary material, Supplemental Table 2. The total count and percentage of 'I do not know' answers are shown in Supplemental Table 3. The associations between children's and parents' reporting are summarized in Table 1 . Kendall's $\tau_{\mathrm{B}}$ correlation between parents' and children's answers ranged from 0.44 for fat meat to 0.81 for puff pastry, indicating medium to large correlations for all foods analysed. Spearman's $\rho$ between parents' and children's answers ranged from 0.55 for sauces and dressings to 0.84 for puff pastry, indicating large correlations for all foods analysed (see Supplemental Table 4). Values of $\kappa$ showed low to high levels of agreement, ranging from 0.15 for sweets to 0.77 for puff pastry. Considering the lower bound of the CI, fair agreement $(\kappa<0 \cdot 20)$ was found for water, fruit, vegetables, starchy foods, meat, dairy products, fats and oils, and sweets. Moderate agreement $(\kappa=0 \cdot 20-0 \cdot 45)$ was found for whole grains, fish, fat meat, sauces and dressings, junk food, sugar drinks and coffee. Strong agreement $(\kappa>0.45)$ was found for eggs, legumes, tofu and quorn, fast food and puff pastry. Finally, Lin's correlation coefficients ranged from 0.39 for whole grains to 0.86 for puff pastry (see Table 1 ).

Kendall's $\tau_{\mathrm{B}}$ correlations between children's and parents' reporting of food by meal are summarized in Table 2 . Kendall's $\tau_{\mathrm{B}}$ values ranged from 0.20 for sugar drinks after dinner to 1.00 for coffee at lunch and dinner, and for eggs after dinner. Vegetables at breakfast, meat at morning snack and puff pastry at afternoon snack showed non-significant results. During some meal occasions, some foods were not 
Table 1 Associations between food items logged by children aged 6-12 years and food items logged by their parents in a week ( $n$ 264); Ticino, Switzerland, April-June 2014

\begin{tabular}{lccccr}
\hline Food item & Kendall's $\tau_{\mathrm{B}}$ & $\kappa$ & $95 \% \mathrm{Cl}$ & Lin's coefficient & $95 \% \mathrm{Cl}$ \\
\hline Water & 0.49 & 0.20 & $0.15,0.25$ & 0.65 & $0.58,0.71$ \\
Fruit & 0.64 & 0.25 & $0.19,0.31$ & 0.77 & $0.72,0.81$ \\
Vegetables & 0.54 & 0.19 & $0.14,0.25$ & 0.59 & $0.53,0.66$ \\
Starchy foods & 0.66 & 0.18 & $0.13,0.23$ & 0.78 & $0.73,0.82$ \\
Whole grains & 0.57 & 0.37 & $0.20,0.54$ & 0.39 & $0.32,0.46$ \\
Meat & 0.47 & 0.23 & $0.17,0.30$ & 0.61 & $0.54,0.68$ \\
Fish & 0.58 & 0.50 & $0.42,0.58$ & 0.79 & $0.74,0.83$ \\
Eggs & 0.64 & 0.58 & $0.50,0.66$ & 0.74 & $0.69,0.79$ \\
Legumes, tofu, quorn & 0.64 & 0.59 & $0.50,0.68$ & 0.75 & $0.69,0.80$ \\
Dairy products & 0.61 & 0.16 & $0.11,0.21$ & 0.73 & $0.67,0.78$ \\
Fats \& oils & 0.52 & 0.17 & $0.11,0.23$ & 0.66 & $0.60,0.73$ \\
Sauces \& dressings & 0.49 & 0.42 & $0.33,0.51$ & 0.56 & $0.48,0.64$ \\
Fat meat & 0.44 & 0.31 & $0.24,0.38$ & 0.53 & $0.45,0.61$ \\
Fast food & 0.58 & 0.55 & $0.47,0.63$ & 0.67 & $0.60,0.73$ \\
Junk food & 0.64 & 0.50 & $0.42,0.58$ & 0.80 & $0.76,0.85$ \\
Sweets & 0.58 & 0.15 & $0.10,0.20$ & 0.71 & $0.65,0.76$ \\
Sugar drinks & 0.59 & 0.32 & $0.26,0.38$ & 0.70 & $0.64,0.76$ \\
Puff pastry & 0.81 & 0.77 & $0.68,0.86$ & 0.86 & $0.84,0.89$ \\
Coffee & 0.62 & 0.56 & $0.36,0.76$ & 0.57 & $0.49,0.65$ \\
\hline
\end{tabular}

All values are significant at $P<0.01$.

Table 2 Associations between food items logged by children aged 6-12 years and food items logged by their parents in a week ( $n$ 264), by meal, using Kendall's $\tau_{\mathrm{B}}$ coefficient; Ticino, Switzerland, April-June 2014

\begin{tabular}{|c|c|c|c|c|c|c|}
\hline \multirow[b]{2}{*}{ Food item } & \multicolumn{6}{|c|}{ Meal } \\
\hline & $\begin{array}{l}\text { Breakfast } \\
\text { (n 264) }\end{array}$ & $\begin{array}{l}\text { Morning snack } \\
\quad(n 261)\end{array}$ & $\begin{array}{l}\text { Lunch } \\
\text { (n 264) }\end{array}$ & $\begin{array}{l}\text { Afternoon snack } \\
\qquad(n \text { 263) }\end{array}$ & $\begin{array}{l}\text { Dinner } \\
(n \text { 262) }\end{array}$ & $\begin{array}{l}\text { After dinner } \\
\quad(n \text { 263) }\end{array}$ \\
\hline Water & 0.78 & 0.31 & 0.45 & 0.56 & 0.48 & 0.60 \\
\hline Fruit & 0.74 & 0.63 & 0.60 & 0.67 & 0.65 & 0.69 \\
\hline Vegetables & $-0.004^{N S}$ & 0.71 & 0.56 & 0.44 & 0.56 & 0.30 \\
\hline Starchy foods & 0.83 & 0.75 & 0.60 & 0.65 & 0.63 & 0.64 \\
\hline Whole grains & 0.58 & 0.71 & 0.31 & 0.82 & 0.54 & NA \\
\hline Meat & 0.65 & $-0.009^{N S}$ & 0.60 & 0.64 & 0.55 & NA \\
\hline Fish & NA & NA & 0.68 & NA & 0.75 & NA \\
\hline Eggs & 0.85 & 0.81 & 0.71 & 0.57 & 0.75 & 1.00 \\
\hline Legumes, tofu, quorn & NA & NA & 0.64 & NA & 0.73 & NA \\
\hline Dairy products & 0.72 & 0.58 & 0.59 & 0.68 & 0.53 & 0.65 \\
\hline Fats \& oils & 0.78 & 0.67 & 0.52 & 0.38 & 0.53 & 0.32 \\
\hline Sauces \& dressings & NA & NA & 0.49 & NA & 0.62 & NA \\
\hline Fat meat & 0.86 & $0 \cdot 70$ & 0.50 & 0.48 & 0.53 & 0.50 \\
\hline Fast food & NA & NA & 0.66 & 0.40 & 0.64 & NA \\
\hline Junk food & 0.69 & 0.76 & 0.47 & 0.74 & 0.41 & 0.60 \\
\hline Sweets & 0.74 & 0.67 & 0.52 & 0.57 & 0.54 & 0.63 \\
\hline Sugar drinks & 0.83 & 0.64 & 0.6 & 0.59 & 0.63 & 0.20 \\
\hline Puff pastry & NA & NA & 0.83 & $-0.004^{N S}$ & 0.86 & NA \\
\hline Coffee & 0.59 & NA & 1.00 & 0.32 & 1.00 & NA \\
\hline
\end{tabular}

NA, food not consumed/sample size too small.

Values are significant at $P<0.01$ except those marked NS.

consumed or the sample size was too small. Hence, the correlation analysis was not performed (e.g. fish and fast food at breakfast and morning snack). Spearman's $\rho$ between children's and parents' reporting of food by meal are summarized in the online supplementary material, Supplemental Table 4.

\section{Association according to gender and grade at school}

For boys and girls, agreement between parents' and children's reporting was similar for most foods. Kendall's
$\tau_{\mathrm{B}}$ correlations between boys and their parents ranged from 0.41 for sauces and dressings to 0.77 for puff pastry, indicating medium to high correlations. Between girls and their parents, Kendall's $\tau_{\mathrm{B}}$ ranged from 0.46 for fat meat to 0.84 for puff pastry, indicating medium to high correlations. Kendall's $\tau_{\mathrm{B}}$ for fruit was 0.65 for boys and 0.63 for girls; for puff pastry it was 0.77 for boys and 0.84 for girls; and for starchy foods it was 0.62 for boys and 0.72 for girls. Kendall's $\tau_{\mathrm{B}}$ correlation coefficients for all food groups according to gender are summarized in Table 3. Spearman's rank correlations are described 
Table 3 Kendall's $\tau_{\mathrm{B}}$ and $\kappa$ values for food items logged by children aged 6-12 years and food items logged by their parents in a week ( $n$ 264), by gender; Ticino, Switzerland, April-June 2014

\begin{tabular}{|c|c|c|c|c|c|c|}
\hline & \multicolumn{2}{|c|}{ Kendall's $\tau_{\mathrm{B}}$} & \multicolumn{4}{|c|}{$\kappa$} \\
\hline & Boys & Girls & Boys & $95 \% \mathrm{Cl}$ & Girls & $95 \% \mathrm{Cl}$ \\
\hline$n$ & 127 & 137 & 127 & - & 137 & - \\
\hline Water & 0.44 & 0.53 & 0.16 & $0.09,0.23$ & 0.24 & $0.17,0.31$ \\
\hline Fruit & 0.65 & 0.63 & 0.21 & $0.13,0.29$ & 0.29 & $0.20,0.38$ \\
\hline Vegetables & 0.45 & 0.61 & 0.18 & $0.10,0.26$ & 0.19 & $0.11,0.27$ \\
\hline Starchy foods & 0.62 & 0.72 & 0.14 & $0.07,0.21$ & 0.22 & $0.14,0.30$ \\
\hline Whole grains & 0.74 & 0.48 & 0.53 & $0.24,0.82$ & 0.28 & $0.08,0.48$ \\
\hline Meat & 0.46 & 0.47 & 0.16 & $0.07,0.25$ & 0.30 & $0.21,0.39$ \\
\hline Fish & 0.54 & 0.63 & 0.44 & $0.32,0.56$ & 0.54 & $0.43,0.65$ \\
\hline Eggs & 0.68 & 0.60 & 0.53 & $0.42,0.64$ & 0.62 & $0.51,0.73$ \\
\hline Legumes, tofu, quorn & 0.67 & 0.62 & 0.65 & $0.51,0.79$ & 0.55 & $0.42,0.68$ \\
\hline Dairy products & 0.61 & 0.60 & 0.13 & $0.06,0.20$ & 0.19 & $0.11,0.27$ \\
\hline Fats \& oils & 0.48 & 0.56 & 0.16 & $0.08,0.24$ & 0.18 & $0.10,0.26$ \\
\hline Sauces \& dressings & 0.41 & 0.57 & 0.39 & $0.25,0.53$ & 0.44 & $0.31,0.57$ \\
\hline Fat meat & 0.43 & 0.46 & 0.27 & $0.17,0.37$ & 0.34 & $0.24,0.44$ \\
\hline Fast food & 0.57 & 0.60 & 0.54 & $0.43,0.65$ & 0.55 & $0.44,0.66$ \\
\hline Junk food & 0.72 & 0.61 & 0.61 & $0.49,0.73$ & 0.41 & $0.30,0.52$ \\
\hline Sweets & 0.60 & 0.57 & 0.11 & $0.05,0.18$ & 0.20 & $0.12,0.28$ \\
\hline Sugar drinks & 0.53 & 0.65 & 0.31 & $0.22,0.40$ & 0.33 & $0.24,0.42$ \\
\hline Puff pastry & 0.77 & 0.84 & 0.73 & $0.58,0.88$ & 0.80 & $0.68,0.92$ \\
\hline Coffee & 0.73 & 0.50 & 0.67 & $0.42,0.92$ & 0.44 & $0.14,0.74$ \\
\hline
\end{tabular}

All values are significant at $P<0.01$.

in the online supplementary material, Supplemental Table 5 .

For boys $\kappa$ values ranged from 0.11 for sweets to 0.73 for puff pastry, while for girls $\kappa$ ranged from $0 \cdot 18$ for fats and oils to 0.80 for puff pastry, showing low to high agreement for both genders. The $\kappa(95 \% \mathrm{CI})$ for sweets was $0 \cdot 11(0 \cdot 05,0 \cdot 18)$ for boys and $0 \cdot 20(0 \cdot 12,0 \cdot 28)$ for girls; for vegetables it was $0.18(0 \cdot 10,0 \cdot 26)$ for boys and 0.19 $(0.11,0.27)$ for girls; for puff pastry it was $0.73(0.58,0.88)$ for boys and $0.80(0.68,0.92)$ for girls; and for fruit it was $0 \cdot 21(0 \cdot 13,0 \cdot 29)$ for boys and $0 \cdot 29(0 \cdot 20,0 \cdot 38)$ for girls. See Table 3 for all food groups according to gender.

Overall, there was higher agreement between children in middle school and their parents, than between children in elementary school and their parents, with few exceptions. Kendall's $\tau_{\mathrm{B}}$ correlations ranged from 0.006 (meat) for children in 1 st grade of elementary school to 1.00 (coffee) for children in middle school. Kendall's $\tau_{\mathrm{B}}$ and $\kappa$ values are summarized in Tables 4 and 5 .

\section{Discussion}

To our knowledge, the present study is one of the few comparing $7 \mathrm{~d}$ food diaries completed by children and parents. Our results showed a significant agreement between children's and parents' reported food consumption; furthermore, the agreement measures tended to be higher than found in the food consumption reporting literature $^{(13)}$. For example, the study conducted in 2004 by Thiagarajah et al. among 120 children attending 4th grade from five elementary schools in south-central Indiana (USA) validated the food behaviour questions from the
School Physical Activity and Nutrition questionnaire ${ }^{(13)}$. The Spearman correlation coefficients are higher in our study compared with Thiagarajah et al. (i.e. $0 \cdot 80 v$. $0 \cdot 40$ for fruit, $0.71 v .0 .52$ for sugar drinks and $0.57 v .0 .43$ for fat/ fried meat, respectively), while the $\kappa$ values are comparable (i.e. $0.25 v \cdot 0.27$ for fruit, $0.32 v \cdot 0.38$ for sugar drinks and $0.31 v \cdot 0.38$ for fat/fried meat, respectively).

Diaries completed by older children and their parents showed higher Spearman correlation coefficients and $\kappa$ values, a finding consistent with previous studies ${ }^{(13,25)}$. While younger children may encounter some difficulties in completing food diaries, several studies have shown that for children older than 8 years of age, validity is higher when the diaries are completed by the child rather than by a proxy ${ }^{(5,9)}$. Further, a validation study conducted among children aged 9-11 years found that mixed foods had lower agreement scores than well-defined foods and foods consumed less frequently ${ }^{(13)}$. Because of the coding system adopted for the present study (i.e. coding each of the foods in a mixed dish separately) our study partially solved this issue.

Studies with adults show women tend to report their dietary intake more accurately than men ${ }^{(26,27)}$; however, in our study, no differences were found between boys and girls and their level of agreement with their parents. Our results are consistent with those reported by Thiagarajah et al., who also found no gender differences in validity, except for a few items (gravy, French fries and chips), where a higher agreement was found in girls ${ }^{(13)}$.

\section{Strengths and limitations}

The methodology used in the current study could serve as reference for other studies assessing children's food intake. First, our analysis included basic food groups, 
Table 4 Kendall's $\tau_{\mathrm{B}}$ values of food items logged by children aged 6-12 years and food items logged by their parents in a week ( $n$ 264), by class at school; Ticino, Switzerland, April-June 2014

\begin{tabular}{|c|c|c|c|c|c|c|c|}
\hline \multirow{2}{*}{$\begin{array}{l}\text { Education } \\
\text { Grade }\end{array}$} & \multicolumn{5}{|c|}{ Elementary school } & \multicolumn{2}{|c|}{ Middle school } \\
\hline & 1st & 2nd & $3 \mathrm{rd}$ & 4th & 5 th & 1st & 2nd \\
\hline$n$ & 26 & 30 & 51 & 60 & 40 & 39 & 15 \\
\hline Water & $0.41^{\star *}$ & $0.52^{\star *}$ & $0.38^{\star *}$ & $0.52^{\star *}$ & $0.71^{\star *}$ & $0.48^{\star *}$ & $0.78^{\star \star}$ \\
\hline Fruit & $0.48^{\star \star}$ & $0.77^{\star \star}$ & $0.63^{\star \star}$ & $0.61^{\star \star}$ & $0.68^{* *}$ & $0.75^{\star *}$ & $0.93^{\star *}$ \\
\hline Vegetables & 0.14 & $0.43^{\star *}$ & $0.57^{* *}$ & $0.59^{\star *}$ & $0.54^{* *}$ & $0.68^{\star *}$ & $0.66^{\star *}$ \\
\hline Starchy foods & $0.50^{\star *}$ & $0.62^{\star \star}$ & $0.70^{\star *}$ & $0.67^{\star \star}$ & $0.61^{* *}$ & $0.80^{\star *}$ & $0.82^{* *}$ \\
\hline Whole grains & NA & NA & $0.83^{\star *}$ & $0.61^{\star *}$ & $0.71^{\star *}$ & $0.39^{\star *}$ & NA \\
\hline Meat & 0.006 & $0.40^{* *}$ & $0.51^{\star *}$ & $0.70^{\star *}$ & $0.36^{* *}$ & $0.65^{\star *}$ & $0.42^{\star \star}$ \\
\hline Fish & 0.29 & $0.40^{\star \star}$ & $0.69^{\star *}$ & $0.50^{\star \star}$ & $0.65^{\star *}$ & $0.71^{\star *}$ & $0.80^{\star *}$ \\
\hline Eggs & $0.39^{*}$ & $0.64^{\star \star}$ & $0.60^{\star *}$ & $0.60^{\star \star}$ & $0.69^{\star \star}$ & $0.81^{\text {** }}$ & $0.76^{\star \star}$ \\
\hline Legumes, tofu, quorn & $0.44^{*}$ & $0.49^{* *}$ & $0.63^{\star *}$ & $0.81^{\star \star}$ & $0.50^{\star *}$ & $0.76^{\star *}$ & 0.37 \\
\hline Dairy products & $0.51^{\star *}$ & $0.50^{\star *}$ & $0.51^{\star *}$ & $0.59^{\star \star}$ & $0.72^{\star \star}$ & $0.74^{\star *}$ & $0.86^{\star *}$ \\
\hline Fats \& oils & $0.35^{\star}$ & $0.45^{\star *}$ & $0.51^{\star *}$ & $0.60^{\star *}$ & $0.55^{\star *}$ & $0.51^{\star \star}$ & $0.53^{* *}$ \\
\hline Sauces \& dressings & 0.16 & 0.29 & $0.68^{* *}$ & $0.49^{\star *}$ & $0.57^{\star \star}$ & $0.56^{\star \star}$ & $0.53^{*}$ \\
\hline Fat meat & 0.20 & $0.39^{\star \star}$ & $0.44^{\star *}$ & $0.48^{\star \star}$ & $0.61^{\star *}$ & $0.53^{\text {** }}$ & $0.75^{\star \star}$ \\
\hline Fast food & $0.42^{\star *}$ & $0.71^{* *}$ & $0.56^{\star \star}$ & $0.49^{\star *}$ & $0.74^{\star *}$ & $0.66^{\star *}$ & $0.73^{\star *}$ \\
\hline Junk food & $0.75^{\star \star}$ & $0.52^{\star \star}$ & $0.60^{\star *}$ & $0.68^{\star \star}$ & $0.61^{\star *}$ & $0.67^{\star \star}$ & $0.87^{\star \star}$ \\
\hline Sweets & $0.30^{\star}$ & $0.52^{\star *}$ & $0.64^{\star *}$ & $0.65^{\star *}$ & $0.65^{\star *}$ & $0.60^{\star *}$ & $0.82^{\star *}$ \\
\hline Sugar drinks & 0.07 & $0.49^{\star *}$ & $0.67^{\star *}$ & $0.61^{\star \star}$ & $0.65^{\star \star}$ & $0.79^{\star *}$ & $0.85^{\star \star}$ \\
\hline Puff pastry & $0.71^{* *}$ & $0.76^{\star *}$ & $0.72^{\star *}$ & $0.93^{\star *}$ & $0.86^{\star *}$ & $0.92^{\star *}$ & $0.79^{\star *}$ \\
\hline Coffee & $0.39^{*}$ & NA & $0.55^{\star *}$ & $0.69^{\star *}$ & $0.54^{\star *}$ & $1.00^{* *}$ & $1.00^{\star *}$ \\
\hline
\end{tabular}

$\mathrm{NA}$, not assessable as no child in the class reported having eaten this food.

${ }^{\star} P<0.05,{ }^{* *} P<0.01$.

Table $5 \kappa$ values of food items logged by children aged $6-12$ years and food items logged by their parents in a week ( $n$ 264), by class at school; Ticino, Switzerland, April-June 2014

\begin{tabular}{|c|c|c|c|c|c|c|c|}
\hline \multirow{2}{*}{$\begin{array}{l}\text { Education } \\
\text { Grade }\end{array}$} & \multicolumn{5}{|c|}{ Elementary school } & \multicolumn{2}{|c|}{ Middle school } \\
\hline & $1 \mathrm{st}$ & 2nd & 3rd & 4th & 5 th & $1 \mathrm{st}$ & 2nd \\
\hline$n$ & 26 & 30 & 51 & 60 & 40 & 39 & 15 \\
\hline Water & 0.04 & $0.13^{\star \star}$ & $0.21^{\star *}$ & $0.19^{* *}$ & $0.21^{\star *}$ & $0.24^{\star *}$ & $0.52^{\star \star}$ \\
\hline Fruit & 0.08 & $0 \cdot 17^{\star \star}$ & $0 \cdot 15^{\star \star}$ & $0 \cdot 19^{\star \star}$ & $0.40^{\star *}$ & $0.38^{\star *}$ & $0.61^{\star *}$ \\
\hline Vegetables & -0.06 & $0.22^{\star \star}$ & $0.18^{\star *}$ & $0 \cdot 18^{\star \star}$ & $0.28^{\star \star}$ & $0.26^{\star *}$ & 0.13 \\
\hline Starchy foods & $0 \cdot 12^{\star \star}$ & $0 \cdot 16^{\star \star}$ & $0 \cdot 11^{\star \star}$ & $0 \cdot 16^{\star \star}$ & $0.22^{\star \star}$ & $0.26^{\star \star}$ & $0.30^{\star \star}$ \\
\hline Whole grains & NA & NA & $0.55^{\star *}$ & $0.42^{\star *}$ & $0 \cdot 66^{\star \star}$ & 0.17 & NA \\
\hline Meat & 0.02 & $0 \cdot 19^{* *}$ & $0 \cdot 12^{\star}$ & $0.30^{\star *}$ & $0 \cdot 19^{\star \star}$ & $0.52^{\star \star}$ & $0.22^{*}$ \\
\hline Fish & 0.09 & $0 \cdot 32^{\star \star}$ & $0.60^{\star *}$ & $0.44^{\star \star}$ & $0.52^{\star \star}$ & $0.69^{\star *}$ & $0.71^{\star \star}$ \\
\hline Eggs & $0.31^{*}$ & $0.37^{* *}$ & $0.68^{* *}$ & $0.49^{* *}$ & $0.61^{\star *}$ & $0.79^{\star *}$ & $0.72^{\star \star}$ \\
\hline Legumes, tofu, quorn & $0.38^{\star *}$ & $0.44^{\star \star}$ & $0.58^{\star \star}$ & $0 \cdot 70^{\star *}$ & $0.45^{\star \star}$ & $0.75^{\star *}$ & 0.32 \\
\hline Dairy products & $0.08^{*}$ & 0.03 & 0.00 & $0.19^{\star *}$ & $0.26^{\star *}$ & $0.32^{* *}$ & $0.21^{* *}$ \\
\hline Fats \& oils & -0.06 & 0.01 & $0.11^{\star}$ & $0.25^{\star \star}$ & $0.23^{\star \star}$ & $0.32^{\star \star}$ & 0.15 \\
\hline Sauces \& dressings & 0.14 & 0.14 & $0.56^{\star *}$ & $0.36^{\star \star}$ & $0.60^{\star *}$ & $0.52^{\star *}$ & $0.52^{\star \star}$ \\
\hline Fat meat & 0.07 & 0.21 & $0.28^{* *}$ & $0.35^{\star *}$ & $0.29^{\star *}$ & $0.44^{\star *}$ & $0.49^{\star \star}$ \\
\hline Fast food & $0.57^{\star *}$ & $0.45^{\star \star}$ & $0.45^{\star \star}$ & $0.50^{\star *}$ & $0.57^{\star \star}$ & $0.75^{\star \star}$ & $0.71^{\star \star}$ \\
\hline Junk food & $0.53^{\star *}$ & $0.44^{\star \star}$ & $0 \cdot 34^{\star *}$ & $0 \cdot 60^{\star *}$ & $0 \cdot 39^{\star \star}$ & $0.53^{\star *}$ & $0.83^{\star *}$ \\
\hline Sweets & 0.07 & 0.04 & $0 \cdot 13^{\star \star}$ & $0 \cdot 14^{\star \star}$ & $0 \cdot 18^{\star \star}$ & $0.21^{\star \star}$ & $0.50^{\star \star}$ \\
\hline Sugar drinks & 0.62 & $0.28^{\star *}$ & $0 \cdot 20^{\star *}$ & $0.33^{\star *}$ & $0 \cdot 32^{\star *}$ & $0.48^{\star *}$ & $0.74^{\star \star}$ \\
\hline Puff pastry & $0.57^{\star *}$ & $0.69^{* \star}$ & $0.64^{\star *}$ & $0.85^{\star *}$ & $0.83^{\star \star}$ & $1.00^{\star *}$ & $0.83^{* *}$ \\
\hline Coffee & $0.37^{\star \star}$ & NA & $0.49^{\star *}$ & $0.48^{\star \star}$ & $0.55^{\star \star}$ & $0.74^{\star *}$ & $1.00^{\star \star}$ \\
\hline
\end{tabular}

$\mathrm{NA}$, not assessable as no child in the class reported having eaten this food.

${ }^{\star} P<0.05,{ }^{\star \star} P<0.01$

which can be obtained for any study across the world. Second, specific cultural foods (i.e. peanut butter, gravy, noodles, etc.) can be easily included in the food categories identified for the study and processed similarly. Furthermore, observation effects were minimized due to the longevity of the data collection, seven consecutive days. One study showed that people typically revert to their normal behaviour after the 'observation effect' wears off in $2-3 \mathrm{~d}^{(28)}$.

The primary limitation in the present study is the lack of a direct observation or biomarkers to compare with selfreporting. This was mostly due to feasibility reasons, as collecting blood or urine samples, or hiring staff to observe families, was far beyond our budget and would have likely 
resulted in a lower participation rate. Further, it cannot be excluded that children and parents completed the food diaries together, even though the importance of completing the diaries separately was stressed in the instructions. The relatively low completion rate of $35 \%$ is unfortunate, but is comparable to participation rates reported elsewhere (16-57\% for men and 31-74\% for women $)^{(29)}$. This is a challenge in food consumption reporting; the $7 \mathrm{~d}$ give a better picture of what children eat than a shorter period reporting, but it also increases respondent burden.

Although $\kappa$ value is commonly used to assess agreement, it might not be appropriate to compare studies, as it depends on the prevalence of the findings and on the number of categories ${ }^{(30-32)}$. Additionally, as the thresholds to define the strength of agreement vary across the literature, our results might have been over- or underestimated. Using the more stringent thresholds by Landis and $\operatorname{Koch}^{(33)}$, the percentage of $\kappa$ values showing moderate agreement increased from $33 \%$ to $43 \%$, while the percentage of $\kappa$ values showing substantial agreement decreased from $38 \%$ to $5 \%$.

We also had to use a non-validated food diary, due to the lack of validated food diaries in Switzerland. Further, social desirability could play a factor in child or parent reporting. Finally, recall bias could not be completely ruled out, but we believe it was minimized due to the requirement that eating behaviour should be recorded immediately after each meal or snack.

\section{Conclusion}

Our study reports significant agreement between children's and parents' reported food consumption, when using $7 \mathrm{~d}$ diaries without reported portion sizes. When assessing children's eating behaviour using a food diary completed each day, children may be just as able as their parents to reliably report what they ate.

\section{Acknowledgements}

Acknowledgements: The authors thank Ms Maria Lagomarsino for her help with data entry. They also thank the parents and children who participated in this study and the teachers, school directors and DECS administration. Financial support: This research received no specific grant from any funding agency in the public, commercial or notfor-profit sectors. Conflict of interest: None. Authorship: Each author contributed equally to the design of this paper, including the research questions, study design, analysis plan and conduct, and writing the manuscript. Ethics of buman subject participation: This study was conducted according to the guidelines laid down in the Declaration of Helsinki and was exempt from full ethics review by the Canton Ticino Ethics Review Committee, in accordance with Swiss Human Subjects Law. Written informed consent was obtained from all participants prior to any data collection.

\section{Supplementary material}

To view supplementary material for this article, please visit http://dx.doi.org/10.1017/S1368980016001488

\section{References}

1. The NS, Suchindran C, North KE et al. (2010) Association of adolescent obesity with risk of severe obesity in adulthood. JAMA 304, 2042-2047.

2. Livingstone MB \& Robson PJ (2000) Measurement of dietary intake in children. Proc Nutr Soc 59, 279-293.

3. Livingstone MBE, Robson PJ \& Wallace JMW (2004) Issues in dietary intake assessment of children and adolescents. Br J Nutr 92, Suppl. 2, S213-S222.

4. Burrows TL, Martin RJ \& Collins CE (2010) A systematic review of the validity of dietary assessment methods in children when compared with the method of doubly labeled water. J Am Diet Assoc 110, 1501-1510.

5. Kolodziejczyk JK, Merchant G \& Norman GJ (2012) Reliability and validity of child/adolescent food frequency questionnaires that assess foods and/or food groups. J Pediatr Gastroenterol Nutr 55, 4-13.

6. McPherson RS, Hoelscher DM, Alexander M et al. (2000) Dietary assessment methods among school-aged children: validity and reliability. Prev Med 31, issue 6, S11-S33.

7. Johnson RK (2002) Dietary intake - how do we measure what people are really eating? Obes Res 10, Suppl. 1, 63S-68S.

8. Johnson-Taylor WL \& Everhart JE (2006) Modifiable environmental and behavioral determinants of overweight among children and adolescents: report of a workshop. Obesity (Silver Spring) 14, 929-966.

9. Collins CE, Watson J \& Burrows T (2010) Measuring dietary intake in children and adolescents in the context of overweight and obesity. Int J Obes (Lond) 34, 1103-1115.

10. Bokhof B, Buyken AE, Doğan C et al. (2012) Validation of protein and potassium intakes assessed from $24 \mathrm{~h}$ recalls against levels estimated from $24 \mathrm{~h}$ urine samples in children and adolescents of Turkish descent living in Germany: results from the EVET! Study. Public Health Nutr 15, 640-647.

11. Frankenfeld CL, Poudrier JK, Waters NM et al. (2012) Dietary intake measured from a self-administered, online 24-hour recall system compared with 4-day diet records in an adult US population. I Acad Nutr Diet 112, $1642-1647$.

12. Wang Y, Beydoun MA, Li J et al. (2011) Do children and their parents eat a similar diet? Resemblance in child and parental dietary intake: systematic review and metaanalysis. J Epidemiol Community Health 65, 177-189.

13. Thiagarajah K, Fly AD, Hoelscher DM et al. (2008) Validating the food behavior questions from the elementary school SPAN questionnaire. J Nutr Educ Behav 40, 305-310.

14. Baranowski T \& Domel SB (1994) A cognitive model of children's reporting of food intake. Am J Clin Nutr $\mathbf{5 9}$, 1 Suppl., 212S-217S.

15. Spook JE, Paulussen T, Kok G et al. (2013) Monitoring dietary intake and physical activity electronically: feasibility, usability, and ecological validity of a mobile-based ecological momentary assessment tool. J Med Internet Res 15, e214. 
16. Fram MS, Frongillo EA, Draper CL et al. (2013) Development and validation of a child report assessment of child food insecurity and comparison to parent report assessment. J Hunger Environ Nutr 8, 128-145.

17. Lim Y, Velozo C \& Bendixen RM (2014) The level of agreement between child self-reports and parent proxyreports of health-related quality of life in boys with Duchenne muscular dystrophy. Qual Life Res 23, 1945-1952.

18. McDougall J, Bedell G \& Wright V (2013) The youth report version of the Child and Adolescent Scale of Participation (CASP): assessment of psychometric properties and comparison with parent report. Child Care Health Dev 39, 512-522.

19. Neuhouser ML, Lilley S, Lund A et al. (2009) Development and validation of a beverage and snack questionnaire for use in evaluation of school nutrition policies. $J$ Am Diet Assoc 109, 1587-1592.

20. Michels KB, Waser M, Ary E et al. (2008) Validation of a questionnaire to assess dietary habits among 5-13-year old school children of farmers and anthroposophic families. J Nutr Environ Med 17, 157-168.

21. Swiss Society for Nutrition (2015) Swiss Food Pyramid. http://www.sge-ssn.ch/it/io-e-te/mangiare-e-bere/modoequilibrato/piramide-alimentare-svizzera/ (accessed May 2015).

22. Cohen J (1988) Statistical Power Analysis for the Behavioral Sciences, 2nd ed. Hillsdale, NJ: Lawrence Erlbaum.

23. Kendall MG \& Gibbons JD (1990) Rank Correlation Methods, 5th ed. New York: Oxford University Press.

24. Munoz SR \& Bangdiwala SI (1997) Interpretation of Kappa and B statistics measures of agreement. J Appl Stat 24, 105-112.
25. Baranowski T, Islam N, Baranowski J et al. (2012) Comparison of a web-based versus traditional diet recall among children. J Acad Nutr Diet 112, 527-532.

26. Yokoyama Y, Sasaki S, Suzukamo Y et al. (2013) Interpersonal psychosocial factors associated with underreported dietary energy intake in hemodialysis patients. J Ren Nutr 23, 37-44.

27. Ovaskainen M-L, Paturi M, Reinivuo H et al. (2007) Accuracy in the estimation of food servings against the portions in food photographs. Eur J Clin Nutr 62, 674-681.

28. Podsakoff PM, MacKenzie SB, Lee JY et al. (2003) Common method biases in behavioral research: a critical review of the literature and recommended remedies. J Appl Psychol 88, 879-903.

29. Tolonen $\mathrm{H}$, Ahonen $\mathrm{S}$, Jentoft $\mathrm{S}$ et al.; European Health Examination Pilot Project (2015) Differences in participation rates and lessons learned about recruitment of participants the European Health Examination Survey Pilot Project. Scand J Public Health 43, 212-219.

30. Hoehler FK (2000) Bias and prevalence effects on kappa viewed in terms of sensitivity and specificity. J Clin Epidemiol 53, 499-503.

31. Viera AJ \& Garrett JM (2005) Understanding interobserver agreement: the kappa statistic. Fam Med 37, 360-363.

32. Chen G, Faris P, Hemmelgarn B et al. (2009) Measuring agreement of administrative data with chart data using prevalence unadjusted and adjusted kappa. BMC Med Res Methodol 9, 5.

33. Landis JR \& Koch GG (1997) The measurement of observer agreement for categorical data. Biometrics 33, 159-174. 